\title{
Front Matter: Volume 10147
}

, "Front Matter: Volume 10147," Proc. SPIE 10147, Optical Microlithography XXX, 1014701 (12 April 2017); doi: 10.1117/12.2277795

SPIE. Event: SPIE Advanced Lithography, 2017, San Jose, California, United States 


\title{
PROCEEDINGS OF SPIE
}

\section{Optical Microlithography XXX}

\author{
Andreas Erdmann \\ Jongwook Kye \\ Editors
}

28 February - 2 March 2017

San Jose, California, United States

Sponsored by

SPIE

Cosponsored by

Cymer, an ASML company (United States)

Published by

SPIE 
The papers in this volume were part of the technical conference cited on the cover and title page. Papers were selected and subject to review by the editors and conference program committee. Some conference presentations may not be available for publication. Additional papers and presentation recordings may be available online in the SPIE Digital Library at SPIEDigitallibrary.org.

The papers reflect the work and thoughts of the authors and are published herein as submitted. The publisher is not responsible for the validity of the information or for any outcomes resulting from reliance thereon.

Please use the following format to cite material from these proceedings:

Author(s), "Title of Paper," in Optical Microlithography XXX, edited by Andreas Erdmann, Jongwook Kye, Proceedings of SPIE Vol. 10147 (SPIE, Bellingham, WA, 2017) Seven-digit Article CID Number.

ISSN: 0277-786X

ISSN: 1996-756X (electronic)

ISBN: 9781510607453

ISBN: 9781510607460 (electronic)

Published by

SPIE

P.O. Box 10, Bellingham, Washington 98227-0010 USA

Telephone +1 3606763290 (Pacific Time) · Fax +1 3606471445

SPIE.org

Copyright (c) 2017, Society of Photo-Optical Instrumentation Engineers.

Copying of material in this book for internal or personal use, or for the internal or personal use of specific clients, beyond the fair use provisions granted by the U.S. Copyright Law is authorized by SPIE subject to payment of copying fees. The Transactional Reporting Service base fee for this volume is $\$ 18.00$ per article (or portion thereof), which should be paid directly to the Copyright Clearance Center (CCC), 222 Rosewood Drive, Danvers, MA 01923. Payment may also be made electronically through CCC Online at copyright.com. Other copying for republication, resale, advertising or promotion, or any form of systematic or multiple reproduction of any material in this book is prohibited except with permission in writing from the publisher. The CCC fee code is 0277-786X/17/\$18.00.

Printed in the United States of America.

Publication of record for individual papers is online in the SPIE Digital Library.

\section{SPIE. DIGITAL \\ SPIEDigitalLibrary.org}

Paper Numbering: Proceedings of SPIE follow an e-First publication model. A unique citation identifier (CID) number is assigned to each article at the time of publication. Utilization of CIDs allows articles to be fully citable as soon as they are published online, and connects the same identifier to all online and print versions of the publication. SPIE uses a seven-digit CID article numbering system structured as follows:

- The first five digits correspond to the SPIE volume number.

- The last two digits indicate publication order within the volume using a Base 36 numbering system employing both numerals and letters. These two-number sets start with 00, 01, 02, 03, 04, 05, 06, 07, 08, 09, OA, OB ... 0Z, followed by 10-1Z, 20-2Z, etc. The CID Number appears on each page of the manuscript. 


\title{
Contents
}

\author{
vii Authors \\ xi Conference Committee
}

\section{PUSHING OPTICAL LIMITS}

1014706 Using heuristic optimization to set SRAF rules [10147-5]

IMAGE AND PROCESS CONTROL

1014709 Process margin improvement through finger-print removal based on scanner leveling data [10147-8]

10147 OA Scanner-to-scanner CD analysis and control in an HVM environment [10147-9]

10147 OC 450mm lithography status for high volume manufacturing [10147-11]

3D RESIST EFFECTS AND MODELING: JOINT SESSION WITH CONFERENCES 10146 AND 10147

10147 OF Experimental characterization of NTD resist shrinkage [10147-14]

10147 OG Investigation of 3D photoresist profile effect in self-aligned patterning through virtual fabrication [10147-15]

LITHO ETCH PROCESS INTERACTION: JOINT SESSION WITH CONFERENCES 10147 AND 10149

$10147 \mathrm{OH}$ Reducing the impact of etch-induced pattern shift on overlay by using lithography and etch tool corrections [10147-16]

10147 Ol Optimal structure sampling for etch model calibration [10147-17]

10147 0J Interlayer verification methodology for multi-patterning processes [10147-18]

\section{COMPUTATIONAL LITHOGRAPHY I}

10147 OL Full chip hierarchical inverse lithography: a solution with perfect symmetry [10147-20]

10147 OM Source defect impact on pattern shift [10147-21]

1014700 Automated detection and classification of printing sub-resolution assist features using machine learning algorithms [10147-23] 
DESIGN INTERACTIONS WITH LITHOGRAPHY: JOINT SESSION WITH CONFERENCES 10147 AND 10148

$101470 Q \quad$ Resist 3D aware mask solution with ILT for hotspot repair [10147-25]

10147 OR Enhanced OPC recipe coverage and early hotspot detection through automated layout generation and analysis [10147-26]

NON-IC APPLICATIONS

10147 OT A physical model for innovative laser direct write lithography [10147-28]

10147 OU Performance analyses of plasmonic lithography [10147-29]

10147 OW Neuroelectronic device process development and challenge [10147-31]

COMPUTATIONAL LITHOGRAPHY II

10147 OY Effective use of aerial image metrology for calibration of OPC models [10147-33]

$10147 \mathrm{OZ}$ Accurate characterization of 2D etch bias by capturing surrounding effects from resist and trench areas [10147-34]

1014711 Si-photonics waveguides manufacturability using advanced RET solutions [10147-36]

\section{OVERLAY OPTIMIZATION}

1014712 Overlay statistics for multiple exposure patterning [10147-37]

1014713 Experimental verification of on-product overlay improvement by intra-lot overlay control using metrology based grouping [10147-38]

1014715 FinFET-induced anisotropy in printing of implantation shapes [10147-40] TOOLINGS

1014716 On-product overlay improvement with an enhanced alignment system [10147-41]

1014717 Reticle heating feed-forward control (RHC2) on NXT:1980Di immersion scanner for enhanced on-product overlay [10147-42]

1014719 The ArF laser for the next generation multiple-patterning immersion lithography supporting green operations and leading edge processes [10147-44]

10147 1A Layout independent leveling (LIL) on NXT:1980Di immersion scanners for enhanced productivity [10147-45] 
10147 1B Immersion lithography scanner resolution performance demonstration on $450 \mathrm{~mm}$ substrates [10147-46]

POSTER SESSION

10147 1E Improving the topography performance of ion implantation resist [10147-48]

$101471 G \quad$ Eliminate the vibration defect for laser interference lithography using an optical chopper system [10147-51]

$10147 \mathrm{1H}$ The pattern-matching based OPC approach for preemptively fixing the weak points [10147-53]

$101471 \mathrm{~J} \quad$ Lithography and OPC friendly triple patterning decomposition method for VIA [10147-55]

$101471 \mathrm{~L}$ The ultra-violet partial coherence modulation transfer function for lithography [10147-57]

$101471 \mathrm{M}$ Constructing freeform source through the combination of neural network and binary ant colony optimization [10147-58]

$101471 \mathrm{~N}$ Development of the next-generation ArF excimer laser with ultra-narrow stable spectral bandwidth for multiple patterning immersion lithography [10147-59]

1014710 Excimer laser gas usage reduction technology for semiconductor manufacturing [10147-60]

10147 IP The thermal aberration analysis of a lithography projection lens [10147-61]

$101471 Q \quad$ Application of optical similarity in OPC model calibration [10147-62]

$101471 \mathrm{R} \quad$ Compact modeling for the negative tone development processes [10147-63]

10147 is Addressing optical proximity correction challenges from highly nonlinear models [10147-64]

10147 1T Alignment solutions on FBEOL layers using ASML scanners [10147-65]

$101471 \mathrm{U} \quad$ Novel methodology to optimize wafer alignment to enhance $14 \mathrm{~nm}$ on product overlay [10147-66]

$101471 \mathrm{~V} \quad$ Process of opto-mechanical design and assembly for reflective mirror subsystem of lithographic projection lens [10147-68]

10147 IW Advanced application of pattern-aware OPC [10147-70] 
1014712 Study of aging behaviour on 193nm phase-shift masks [10147-74]

1014720 Image acquisition and motion positioning system design based on the projection lens wavefront aberration measurement [10147-75] 
Huang, Jiun-Woei, $1 \mathrm{~L}$

Hutchinson, Trent, 12

Hwang, Hyunwoo, 17, 1A

Ichinose, Go, 16

Isoyan, Artak, OM

Jaenen, Patrick, $\mathrm{OH}$

Jang, Jong Hoon, 17, $1 \mathrm{~A}$

Jang, Se-Yeon, 09, 0A, 17

Jang, Stephen, is

Jehoul, Christiane, $\mathrm{OH}$

Jobes, Mark, 00

Jongen, Joris, 17

Jung, Sunwook, $0 \mathrm{~J}$

Kallingal, Chidam, 06

Kamimura, Sou, OF

Kang, Young Seog, 09, 0A, 17, 1A

Kerrien, G., 11

Khalek, Samah, 1 A

Khikhlovskyi, Seva, $1 \mathrm{~T}$

Kim, Chung-Yong, 09, 0A

Kim, HyoungKook, OC, 1B

Kim, Jinhee, OJ

Kim, Jungmin, OJ

Kim, Young Ha, 17, 1 A

Kim, Young Jun, 09

Klostermann, Ulrich, OF

Kohli, Kriti K., 00

Kong, Jeong-Heung, 09, 0A, 17, 1A

Kou, Weitian, 13

Kubis, Michael, $\mathrm{OH}$

Küchler, Bernd, OF

Kumazaki, Takahito, 19, 1N

Kuo, Fred, IR

Kuo, Hung-Fei, $1 M$

Kupers, Michiel, 13

Kuroda, Takuya, 0C, 1B

Kurosu, Akihiko, 19, 1N, 10

Lafferty, Neal, OR

Lai, Nelson, IW

Lecarpentier, Laurent, 12

Lee, Byeong Soo, 17, 1A

Lee, Honggoo, 13

Lee, Shawn, $1 \mathrm{U}$

Leray, Philippe, $\mathrm{OH}$

Leslie, Alan, 06

Li, Bing, 20

Li, Liang, $1 \mathrm{~J}$

Li, Sikun, IP

Li, Waikin, OF

Li, Zhao-Ze, OA

Liang, Gaofeng, OU

Lie, Frederick, $1 \mathrm{M}$

Lin, Lawrence, IW

Lin, Te-Hsun, $1 G$

Lin, Wei-Cheng, IV

Lin, Yu-Chuan, 1V

Liv, Guangyi, 20

Liu, Jian, 06

Liu, Qingwei, 1J

Liubich, V., 11
Lutich, Andrey, 0 I

Madkour, Kareem, OR

Mai, Hsuan-Ying, IG

Mai, Yung-Ching, IW

Mali, Rajan, IT

Mao, Yanjie, IP

Matsunaga, Takashi, 19, 1N, 10

McGinty, Chris, OR

Meiring, Jason, OR

Melvin, Lawrence S., III, OM, IR

Minghetti, Blandine, 1T, $1 \mathrm{U}$

Miyamoto, Hirotaka, 19

Mizoguchi, Hakaru, 19, 1N, 10

Moe, Andrew, 09, 0A

Moest, Barry, 17

Moon, Junghwan, 13

Morita, Masahiro, 16

Mülders, Thomas, OF

Munson, Jasper P., OC, 1B

Nam, Youngsun, 17, 1A

Nikolsky, Peter, OA

Oh, Seung Chul, $1 \mathrm{U}$

Ohta, Takeshi, 19, 1N, 10

Okamoto, Takanobu, 16

Onanuga, Temitope, OT

Oouchi, Takuma, 10

Orlando, B., 11

Oti, Elliot, IA

Ouyang, Ruiyue, 09

Paarhuis, Bart, 17

Pap, Andras, OG

Park, Noh-Kyoung, OA

Park, Sejin, OJ

Park, Tony, 09, 0A

Peng, Austin, OA

Perez, Victor A., OC, 1B

Pogliani, Carlo, $1 Z$

Poonawala, Amyn, 1s

Qi, Yuejing, 20

Raas, Marcel, 1A

Rangarajan, Balaji, 09

Robinson, Matthew, OW

Rumler, Maximilian, OT

Rutten, Jeroen, 09

Ryu, Machi, OA

Samudrala, Pavan, 1T, 1U

Sawh, Chander, OM

Schatz, Jirka, ol

Schmoeller, Thomas, $O Q$

Schumacher, Dan, OR

Selinidis, Kosta, $0 Q$

Shiba, Yuji, 16

Shibazaki, Yuichi, 16

Shin, Jangho, OA

Shin, Ju Hee, OA

Shirakawa, Michihiro, OF

Simons, Geert, $\mathrm{OH}$

Slaughter, Gymama, OW

Slotboom, Daan, 13

Sobieski, Daniel, $\mathrm{OH}$ 
Song, Changrock, 13

St. John, Matt, is

Stavenga, Marco, 17

Strolenberg, Chris, OA

Sturtevant, John, OJ, OY

Su, Xiaojing, $1 \mathrm{E}$

Subramany, Lokesh, 1T, IU

Sun, Gang, 1P

Sundermann, Frank, $1 Z$

Sung, Yun-A, OA

Taoka, Hironobu, OF

Tejnil, Edita, $1 Q$

Thaler, Thomas, OY

Thivolle, Nicolas, $1 \mathrm{Z}$

Tomasello, Vito, OA

Tritchkov, A., 11

Tseng, Shih-Feng, $1 \mathrm{~V}$

Tsushima, Hiroaki, 19, 1N, 10

Tu, Yuanying, $1 \mathrm{~J}$

Tyson, Joel, OW

Ur-Rehman, Samee, $\mathrm{OH}$

van Delft, Jan-Pieter, 09

van der Wielen, Jeroen, 17

van Hoof, Bram, IA

van Kemenade, Jan, IA

Verbeek, Niek, 17

Viatkina, Katja, $\mathrm{OH}$

von Sydow, Axel, 09

Voncken, Maarten, 1A

Wang, ChangAn, 06

Wang, Jen-Shiang, $\mathrm{OZ}$

Wang, Jian, IP

Wang, Jinze, $0 Z$

Wang, Xiangzhao, IP

Wang, Xiren, 15

Wei, Yayi, $1 \mathrm{E}$

Weichselbaum, Stefan, 17, 1A

Weisbuch, François, 01

Wilkinson, William, 06

Wise, Rich, $\mathrm{OH}$

Woo, Jaesun, 13

Woo, Youngseok, 15

Xiao, Guangming, $0 Q$

Yamamoto, Hajime, 16

Yan, Guanyong, 1J

Yang, Yin-Kuang, $1 G$

Yashiro, Masanori, 10

Ye, Jun,

Yeh, Shin-Shing, IW

Yenikaya, Bayram, OL, IW

Yesilada, E., 11

Yoshidome, Masahiro, OF

Ypma, Alexander, 13

Yu, Hyun-Woo, 13

Zeggaoui, N., 11

Zhang, Chen J., OW

Zhang, Wenzhe, $\mathrm{OH}$

Zhang, Yunqiang, is

Zhao, Qiao, 0 Z

Zheng, Leiwu, $0 Z$
Zhu, Alan, IW

Zuniga, Christian, 15 


\title{
Conference Committee
}

\author{
Symposium Chair
}

Bruce W. Smith, Rochester Institute of Technology (United States)

Symposium Co-chair

Will Conley, Cymer, An ASML company (United States)

Conference Chair

Andreas Erdmann, Fraunhofer-Institut für Integrierte Systeme und Bavelementetechnologie IISB (Germany)

Conference Co-chair

Jongwook Kye, GLOBALFOUNDRIES Inc. (United States)

Conference Program Committee

Pary Baluswamy, Micron Technology, Inc. (United States)

Will Conley, Cymer, An ASML company (United States)

Jo Finders, ASML Netherlands B.V. (Netherlands)

Carlos Fonseca, Tokyo Electron America, Inc. (United States)

Tsai-Sheng Gau, Taiwan Semiconductor Manufacturing Company Ltd. (Taiwan)

Bernd Geh, Carl Zeiss SMT Inc. (United States)

Yuri Granik, Mentor Graphics Corporation (United States)

Harsha Grunes, Intel Corporation (United States)

Young Seog Kang, SAMSUNG Electronics Company, Ltd.

(Korea, Republic of)

Sachiko Kobayashi, Toshiba Corporation (Japan)

Kafai Lai, IBM Corporation (United States)

Michael Liehr, SUNY CNSE/SUNYIT (United States)

Soichi Owa, Nikon Corporation (Japan)

Ryan Pearman, D2S, Inc. (United States)

John S. Petersen, Periodic Structures, Inc. (United States)

Mark C. Phillips, Intel Corporation (United States)

Daniel Sarlette, Infineon Technologies Dresden (Germany)

Xuelong Shi, Semiconductor Manufacturing International Corporation (China)

Bruce W. Smith, Rochester Institute of Technology (United States)

Kazuhiro Takahashi, Canon Inc. (Japan)

Geert Vandenberghe, IMEC (Belgium) 
Reinhard Voelkel, SUSS MicroOptics SA (Switzerland)

Uwe D. Zeitner, Fraunhofer-Institut für Angewandte Optik und

Feinmechanik (Germany)

\section{Session Chairs}

1 Keynote Session

Andreas Erdmann, Fraunhofer-Institut für Integrierte Systeme und

Bavelementetechnologie IISB (Germany)

Jongwook Kye, GLOBALFOUNDRIES Inc. (United States)

2 Pushing Optical Limits

Geert Vandenberghe, IMEC (Belgium)

Young Seog Kang, SAMSUNG Electronics Company, Ltd.

(Korea, Republic of)

3 Image and Process Control

Soichi Owa, Nikon Corp. (Japan)

Andreas Erdmann, Fraunhofer-Institut für Integrierte Systeme und Bavelementetechnologie IISB (Germany)

4 3D Resist Effects and Modeling: Joint Session with Conferences 10146 and 10147

Carlos Fonseca, Tokyo Electron America, Inc. (United States)

Rick Uchida, Tokyo Ohka Kogyo America, Inc. (United States)

5 Litho Etch Process Interaction: Joint Session with Conferences 10147

and 10149

Richard J. Wise, Lam Research Corporation (United States)

Yuri Granik, Mentor Graphics Corporation (United States)

6 Computational Lithography I

John S. Petersen, Periodic Structures, Inc. (United States)

Kafai Lai, IBM Corporation (United States)

7 Design Interactions with Lithography: Joint Session with Conferences

10147 and 10148

Daniel Sarlette, Infineon Technologies Dresden (Germany)

Luigi Capodieci, KnotPrime Inc. (United States)

8 Non-IC Applications

Reinhard Voelkel, SUSS MicroOptics SA (Switzerland)

Andreas Erdmann, Fraunhofer-Institut für Integrierte Systeme und Bavelementetechnologie IISB (Germany) 
9 Computational Lithography II

Ryan Pearman, D2S, Inc. (United States)

Harsha Grunes, Intel Corporation (United States)

10 Overlay Optimization

Sachiko Kobayashi, Toshiba Corporation (Japan)

Xuelong Shi, Semiconductor Manufacturing International Corporation (China)

11 Toolings

Bernd Geh, Carl Zeiss SMT Inc. (United States)

Kazuhiro Takahashi, Canon Inc. (Japan)

12 Latest News

Mark C. Phillips, Intel Corporation (United States)

Bernd Geh, Carl Zeiss SMT Inc. (United States) 